\title{
The use of stem cells for treatment of premature ovulation failure and generation of oocytes
}

\begin{abstract}
Background: Premature Ovarian Failure (POF) is a life-changing condition that affects $1 \%$ of women under the age of 40 . POF is also known as premature menopause, and hyper gonadotropic hypogonadism; POF can be primary (spontaneous) or secondary which is usually induced by radiation, chemotherapy or surgery. It comprises of amenorrhea, high menopausal levels of gonadotropin and sex steroid deficiency. The use of mesenchymal stem cells in treatment of POF has been reviewed using different sources of MSCs of umbilical cord, bone marrow transplantation, amniotic fluid and adipose tissue. Many authors have highlighted the importance of re-establishment of hormone or cytokine profiles supporting stem cell follicular development is crucial for the improvement of both the damaged ovary function and fertility recovery.
\end{abstract}

Objective: We are proposing to establish an animal model with induced POF after which MSC is transplanted to reverse POF.

Materials and methods: This can eventually be applied to humans with the use of antilogous or HLA matching of MSCs transplantation, using two sources of bone marrow and adipose tissue MSCs for regeneration of oocytes and thus the reversal of premature ovarian failure.

Results: A proposal of this study and later the execution will help open the door many patients with premature ovarian failure.

Conclusion: This will eventually lead to the importance of studying the global gene expression in human female with POF verses healthy ones.

Keywords: premature ovarian failure, stem cells, mesenchymal cells, infertility, adipose tissue

\author{
Special Issue - 2018
}

\section{Dania H Al Jaroudi,' Dalia A Ali ${ }^{2}$}

'Department of Reproductive Endocrine and Infertility,Women's Specialized Hospital, Saudi Arabia

2Department of Anatomy, King Saud University, Saudi Arabia

Correspondence: Dania H Al Jaroudi, Consultant Obstetrics and Gynecology, Reproductive Endocrine and Infertility, Minimally Invasive Gynecologic Surgery, Department of Reproductive Endocrine and Infertility, Women's Specialized Hospital, King Fahad Medical City, PO Box 59046, Riyadh || 525, Saudi Arabia, Tel +966-| -288-9999 ext I2 | 100, Fax +966-I-288-9999 ext: 3714,

Email daljaroudi@kfmc.med.sa, dania@dohaim.com

Received: March 0I, 2017 | Published: November 30, 2018

\section{Introduction}

Premature Ovarian Failure (POF) is a life-changing condition that affects $1 \%$ of women under the age of 40 . POF is also known as premature menopause, and hypergonadotropic hypogonadism, POF can be primary (spontaneous) or secondary which is usually induced by radiation, chemotherapy or surgery. It comprises of amenorrhea, high menopausal levels of gonadotropin and sex steroid deficiency. Possible causes of premature ovarian failure include genetic and non-genetic causes. Genetic causes such as X linked, autosomal disorders as in myotonia dystrophica, FSH receptor polymorphism, Galactosemia, Inhibin B polymorphism, abnormal Gonadotropin, enzyme deficiencies, signal defects, including abnormal G proteinHLA-DR3 linked predisposition to POF BEPI syndrome have been described. The Non-genetic causes include iatrogenic extirpation of the gonads: surgery/irradiation/chemotherapy, immune diseases such as congenital thymic aplasia/polyglandular failure syndromes I \& II, toxins like smoking, oophritis, or other idiopathic causes. ${ }^{2}$ Cyclophosphamide involves the greatest risk for inducing ovarian failure among all chemotherapeutic agents (odds ratio 3.98 in comparison with unexposed patients). ${ }^{3,4}$ Stem cells are cells with the capacity for prolonged or unlimited self-renewal, it have the capacity to develop into at least one type of highly differentiated cell type. Stem cells are classified mainly to Pluripotent as embryonic stem cells (ES), which are derived from totipotent cells of the early mammalian embryo and are capable of unlimited, undifferentiated proliferation in vitro $^{5}$ and Multipotent stem cells i.e. mesenchymal stem cells (MSCs) that can be induced to differentiate into adipocytes, chondrocytes and osteocytes under appropriate conditions. ${ }^{6}$ In 2006, Takahashi and Yamanaka demonstrated that pluripotent stem cells (IPS) can be generated from mouse embryonic or adult fibroblast by generated by introducing four factors, Oct3/4, Sox2, c-Myc, and Klf4, under ES cell culture conditions, those cells generated exhibit the morphology and growth properties of ES cells and express ES cell marker genes. The use of Es and IPS have the ability to direct them into any cell type for therapeutic potentials and target clinical regenerative medicine yet they still hold limitations in use in clinical therapy because of ethical, Federal regulations and high risk of teratoma formation in vitro. $^{7-9}$ MSC are investigated in a number of clinical trials for tissue repair e.g. bone and cartilage defects, for the enhancement of tissue regeneration e.g. myocardial infarction, and immune modulation e.g. graft-versus-host disease (GvHD) ${ }^{10-12}$ They can be isolated from adipose tissue, umbilical cord and skin. ${ }^{13}$ Placenta $^{14}$ and teeth. ${ }^{15}$ The use of mesenchymal stem cells in treatment of POF have been reviewed and demonstrated by Dan et al. ${ }^{16}$ Using different sources of MSCs of umbilical cord, bone marrow transplantation, amniotic fluid and adipose tissue. The author has highlighted the importance of re-establishment of hormone or cytokine profiles supporting stem cell follicular development is crucial for the improvement of both the damaged ovary function and fertility recovery. ${ }^{16}$ An animal study in Egypt was performed by Gabr et al. the researchers have induced POF in mice and ovulation was restored after MSC treatment. This was 
also demonstrated in a human clinical trial of 20 cases with premature ovarian failure where autologous bone marrow derived MSC were injected in to the ovaries laparoscopically; evidence of ovulation was observed in 5 out of 20 patients. In another study, human endometrial stem cells (HuMenSCs) were injected into a mouse model of POF and demonstrated the safety in vivo use of MSCs in treatment of POF. ${ }^{17}$ Somia et al. demonstrated that intravenous injection of MSCs into rabbits with chemotherapy-induced ovarian damage improved ovarian function. ${ }^{18}$ Song et al. ${ }^{19}$ \& Wang et al. ${ }^{20}$ and had in rat model used umbilical cord derived MSCs to reverse the POF. ${ }^{19,20}$ Sun highlighted the successful use of adipose derived MSC in POF - mouse model; cells were transplanted either directly into bilateral ovaries or via intravenous injection. ${ }^{21}$ Hershlag et al. \& Salooja et al. ${ }^{22,23}$ reported similar successful results in human clinical stud; two patients with Hodgkin's disease and two with advanced breast carcinoma received autologous bone marrow or peripheral blood stem cells transplant, the treatment reversed their menopause signs and all four established pregnancies. ${ }^{22,23}$ The Phenotype identity and genetic changes of MSC will determine the differentiation potentials into certain cell types by the cross talk of the signaling pathway, transcriptional regulatory network, chromatin remodeling and non-coding RNA and epigenetic changes. ${ }^{24}$ The study of global gene expression in MSCs improved the understanding of the regulation of ovarian function. MicroRNA (miRNAs) of miR-23a was upregulated in the plasma of POF while miR-29a and miR-144 are downregulated in POF tissues ${ }^{25}$ and mutation in the FOXL2 caused POF. ${ }^{26,27}$

We are proposing to establish an animal model with induced POF after which MSC is transplanted to reverse POF. This can eventually be applied to humans with the use of autologous or HLA matching of MSCs transplantation, using two sources of bone marrow and adipose tissue MSCs for regeneration of oocytes and thus the reversal of premature ovarian failure. This will eventually lead to the importance of studying the global gene expression in human female with POF verses healthy ones. This study can be done in an animal hospital-based facility with sufficient resources to direct and perform such a study. Therefore, female (mice, rat or rabbit) can be given chemotherapy, Cyclophosphamide to induce premature menopause. Female fertile animal will then be tested for ovarian function and then will be given chemotherapy in the form of Cyclophosphamide to induce premature menopause. This will be followed by injecting MSCs to generate oocytes. After housing with males, the number of pregnancies and offspring will be checked. The main outcome measure will be the regeneration of oocytes after injecting mesenchymal stem cells will lead the way to start the human clinical trials. Using human blood RNA from POF female vs healthy ones, global gene expression can be studied to highlight more genes and MiRNAs involves in the pathogenesis POF.

\section{Conclusion}

This study should be conducted in an academic institute whereby it will help answer one of many unanswered questions in cases of premature ovarian failure, where regeneration of oocytes and the secretion of estrogen can induce a functioning oocyte after its destruction by chemotherapy and genetic diseases with the help of the stem cells.

\section{Acknowledgements}

None.

\section{Conflict of interest}

The author declares no conflict of interest.

\section{References}

1. Panay N, Kalu E. Management of premature ovarian failure. Best Pract Res Clin Obstet Gynaecol. 2009;23(1):129-140.

2. Palo L, Santoro N. Premature ovarian failure (POF): discordance between somatic and reproductive aging. Ageing Res Rev. 2002;1(3):413423

3. Meirow D. Ovarian injury and modern options to preserve fertility in female cancer patients treated with high dose radiochemotherapy for hemato-oncological neoplasias and other cancers. Leuk Lymphoma. 1999;33(1-2):65-76.

4. Dyce P, Wen L, Li J. In vitro germline potential of stem cells derived from fetal porcine skin. Nature Biotechnology. 2006;8(4):384-390.

5. Thomson JA, Itskovitz-Eldor J, Shapiro SS, et al. Embryonic Stem Cell Lines Derived from Human Blastocysts. Science. 1998;282(5391):11451147.

6. Pittenger MF, Mackay AM, Beck SC, et al. Multilineage potential of adult human mesenchymal stem cells. Science. 1999;284(5411):143147.

7. de Wert G, Mummery C. Human embryonic stem cells: research, ethics and policy. Hum Reprod. 2003;18(4):672-682.

8. Takahashi K, Yamanaka S. Induction of Pluripotent Stem Cells from Mouse Embryonic and Adult Fibroblast Cultures by Defined Factors. Cell. 2006;126(4):663-676.

9. Puri MC, Nagy A. Concise Review: Embryonic Stem Cells Versus Induced Pluripotent Stem Cells: The Game Is On. Stem Cells. 2012;30(1):10-14.

10. Kassem M, Al Nbaheen M, Ali D, et al. Human Mesenchymal (skeletal) stem cells and clinical applications for bone tissue regeneration. In: Bongso A, Lee EH, editors. Stem Cells: From bench to bedside. 2nd ed. World Scientific publishing co. pte Ltd 2, Singapore; 2010. p. 457-475.

11. Abdallah BM, Kassem M. Human mesenchymal stem cells: from basic biology to clinical applications. Gene Therapy. 2008;15(2):109-116.

12. Le Blanc K, Frassoni F, Ball L, et al. Mesenchymal stem cells for treatment of steroid-resistant, severe, acute graft-versus-host disease: a phase II study. Lancet. 2008;371(9624):1579-1586.

13. Vishnubalaji R, Al Nbaheen M, Kadalmani B, et al. Comparative investigation of the differentiation capability of bone-marrow-and adipose-derived mesenchymal stem cells by qualitative and quantitative analysis. Cell Tissue Res. 2012;347(2):419-427.

14. Miao X, Wu X, Shi W. Umbilical cord mesenchymal stem cells in neurological disorders: A clinical study. Indian J Biochem Biophys. 2015;52(2):140-146.

15. Perry BC, Zhou D, Wu X, et al. Collection, cryopreservation, and characterization of human dental pulp-derived mesenchymal stem cells for banking and clinical use. Tissue Eng Part C Methods. 2008;14(2):149456.

16. Dan S, Haibo L, Hong L. Pathogenesis and stem cell therapy for premature ovarian failure. OA Stem Cells. 2014;2(1):4-8

17. Liu T, Huang Y, Zhang J, et al. Transplantation of Human Menstrual Blood Stem Cells to Treat Premature Ovarian Failure in Mouse Model. Stem Cells Dev. 2014;23(13):1548-1557. 
18. Abd Allah SH, Shalaby SM, Pasha HF, et al. Mechanistic action of mesenchymal stem cell injection in the treatment of chemically induced ovarian failure in rabbits. Cytotherapy. 2013;15(1):64-75.

19. Song D, Zhong Y, Qian C, et Al. Human Umbilical Cord Mesenchymal Stem Cells Therapy in Cyclophosphamide-Induced Premature Ovarian Failure Rat Model. Biomed Res Int. 2016;2016:2517514.

20. Wang S, Yu L, Sun M, et al. The Therapeutic Potential of Umbilical Cord Mesenchymal Stem Cells in Mice Premature Ovarian Failure. Biomed Res Int. 2013;2013:690491.

21. Sun M, Wang S, Li Y, et al. Adipose-derived stem cells improved mouse ovary function after chemotherapy-induced ovary failure. Stem Cell Res Ther. 2013;4(4):1-80.

22. Hershlag A, Schuster MW. Return of fertility after autologous stem cell transplantation. FertilSteril. 2002;77(2):419-421.
23. Salooja N, Szydlo RM, Socie G, et al. Pregnancy outcomes after peripheral blood or bone marrow transplantation: a retrospective survey. The Lancet. 2001;358(9278):271-276.

24. Ali D, Hamam R, Alfayez M, et al. Epigenetic Library Screen Identifies Abexinostat as Novel Regulator of Adipocytic and Osteoblastic Differentiation of Human Skeletal (Mesenchymal). Stem Cells Transl Med. 2016;5(8):1036-1047.

25. Li Y, Fang Y, Liu Y, et al. MicroRNAs in ovarian function and disorders. J Ovarian Res. 2015;8:1-51.

26. Harris SE, Chand AL, Winship IM, et al. Identification of novel mutations in FOXL2 associated with premature ovarian failure. Mol Hum Reprod. 2008;8(8):729-733.

27. Uda M, Ottolenghi C, Crisponi L, et al. Foxl2 disruption causes mouse ovarian failure by pervasive blockage of follicle development. Hum Mol Genet. 2004;13(11):1171-1181. 